\title{
Mining Logomaps for Ecosystem Intelligence
}

\author{
Rahul C. Basole, PhD \\ Accenture AI \\ rahul.basole@accenture.com
}

\begin{abstract}
Ecosystem intelligence is typically based on highly structured data. More recently, we have seen a growth in extracting knowledge from unstructured textual data sources. Yet, one form of unstructured data has largely been ignored in ecosystem intelligence: image-based data. With an increased use of images and graphics in corporate presentations, social media posts, and annual reports, there is a greater need and opportunity to mine this potentially trapped knowledge. We introduce and describe a human-assisted knowledge discovery approach applied to one particular type of image-based data, namely logomaps, combining image recognition, graph modeling, and visualization to provide insights into business ecosystems. We demonstrate the logomap mining method through a case study of the emerging artificial intelligence (AI) ecosystem and conclude with a discussion of implications and future work.
\end{abstract}

\section{Introduction}

Ecosystems play an important role in the value-creating activities of companies [1, 2]. Ecosystems are highly dynamic systems, with new actors, segments, and relationships emerging and dissolving constantly [3]. Companies in seemingly distant industries can become disruptors over time [4]. Keeping track and making sense of ecosystems is thus paramount to survival, growth, and success [5].

Yet, the scale, speed, and complexity of business ecosystems can make this task quite challenging. Past approaches typically included resource-intensive analyses or subscription to expensive third-party market research reports. However, these approaches have limitations as they are typically static snapshots, inflexible to custom exploration and discovery, and not scalable, thus failing to appropriately capture and portray the complex evolving structure of business ecosystems [5]. To address this issue, researchers started leveraging computational techniques applied to a wider set of ecosystem data and communicating the resulting insights visually $[6,7,8]$. Over time, the data focus has shifted from highly curated datasets to semi- and then unstructured textual data, such as press releases, analyst reports, investor transcripts, news, and blogs [9]. However, despite their pervasiveness and content-richness, image-based data have yet to be considered in business ecosystem intelligence tasks.

In this paper, we introduce and describe a human-assisted knowledge discovery approach applied to one particular type of image-based data relevant to ecosystem intelligence, namely logomaps. A logomap is a composite infographic consisting of corporate logos, market segments, and logical hierarchies, typically created by investors and analysts, that aims to communicate complex, emerging industries to a broader audience. Logomaps often contain a wealth of information on the actual, perceived, and speculated structure of markets, which if decrypted, could provide novel ecosystem insights. They are typically found in reports, corporate presentations, and social media posts. While humans can digest information contained in a single logomap rather rapidly, making sense of a collection of logomaps and extracting systematic knowledge from them is difficult and resource-intensive. We posit that a human plus machine logomap mining approach, consisting of image recognition, graph modeling, and network visualization, can advance our understanding of ecosystems and contribute to the broader field of data mining for business analysis [10]. We demonstrate the value and utility of the logomap mining method through a case study of the emerging artificial intelligence (AI) ecosystem and conclude with research implications and future work.

\section{Related Work}

\subsection{Ecosystem Intelligence}

The study of ecosystems has been a topic of continuously growing interest across many disciplines for many years $[11,12]$. Some of the key tenets 
of the ecosystem conceptualization is that actors are interconnected, co-dependent, and assume specific roles (platform, niche, complementors) in value creating activities [1]. While early studies have focused predominantly on understanding the "what" and "how", more recent work has started to explore the "why" and "when" of ecosystems [13]. Indeed, the structure and dynamics of ecosystems has been examined extensively across a wide range of industry contexts [14].

Methodologically, studies have typically employed conceptual, case study, and empirical approaches. Within these studies, the unit(s) of analysis can vary widely, from firms and people to products and technologies. The central premise of ecosystem intelligence is to shed light into the organization and behavior of existing and emerging ecosystems, providing systemic, group, and actor-level insights and decision support [15]. The growth in empirical ecosystem research can be in particular explained by the broader availability of a wide range of data [9]. Most existing ecosystem intelligence studies leverage structured data sources. More recently, and to answer questions of why and when, researchers have turned to unstructured textual data (such as business description, press releases, industry reports).

Given the scale and complexity, many data-driven intelligence studies utilize interactive visual analytics approaches to explore and explain ecosystem phenomena [7]. In these studies, ecosystems are frequently modeled as complex networks, with nodes representing entities of interest and links denoting explicit or derived relationships, activities, or associations [16]. Pending the fundamental questions being asked, visualization layouts and encodings can include force-directed graphs, geographic maps, hierarchical structures, or flow diagrams.

This study extends prior work by (1) mining a novel type of data (i.e., image-based data) using an integrative human-machine approach, (2) constructing a corresponding knowledge graph, and (3) visualizing the resulting structure of the ecosystem.

\subsection{Image Mining}

Humans can see, rapidly process, and act on visual input [17]. Indeed, vision is one of the most powerful human senses, and one that is extremely difficult to replicate in machines. Recent advances in computer vision, a sub-field of artificial intelligence, facilitated by significant leaps in machine learning technology, computing power, data storage, and high-quality inexpensive input devices, are changing that rapidly [18]. Computer vision enables machines to classify, identify, verify, and detect objects in image-based data and understand, analyze and extract meaningful data, often faster and more accurately than humans.

There are many applications of computer vision in business today including facial recognition for security and surveillance, monitoring inventory or tracking customers in retail, enabling autonomous vehicles by identifying road signs and pedestrians to determine what action to take, diagnosing disease and other ailments in healthcare, or in marketing to identify and target customers with specific brands affinities [19]. The majority of these applications are generally for real-time use. The use of computer vision for more "slow" analytics questions needed in business strategy and intelligence, however, seems limited.

\subsection{Logomaps}

A logomap, also frequently referred to as a market map or an industry landscape, can be described as an image that contains rich logo-based information on a range of relevant ecosystem entities (i.e., companies, products, technologies) and their relationships (see Figure 1). Logomaps are generated by a wide range of creators, including market research firms, investors, as well as individual analysts, with the purpose of describing existing and emerging industries in a visually pleasing manner. Given their ability to summarize complex market contexts with potentially more recognizable and memorable logos [20], logomaps can often be found in corporate presentations, websites, and social media. The increasing popularity of using such representations has even led to the emergence of automated logomap creation tools, such as those offered by CBInsights ${ }^{1}$ or PitchBook ${ }^{2}$.

Given the high degree of freedom in designing logomaps, there is no single unifying template that describes them all. Many variations can exist, including size, orientation, and background. Yet, some common design patterns exist. Following principles of Gestalt psychology, for instance, logomaps often leverage proximity, orientation, and coloring of logos and labels to denote grouping [21]. Logomaps also often use explicit or visually implicit shapes/boxes/borders to denote groups and hierarchies. Most logomaps contain a title, describing the context as well the name of the creator (individual or firm). Unfortunately, logomaps can also contain lots of chart-junk and annotations.

What complicates mining of logomaps further is the diverse options of logos that are at the creator's disposal for any given ecosystem entity. Logos

\footnotetext{
${ }^{1}$ https://www. cbinsights.com/market-map-maker

${ }^{2}$ https://pitchbook.com/products/desktop/ discovery/market-maps-tool
} 


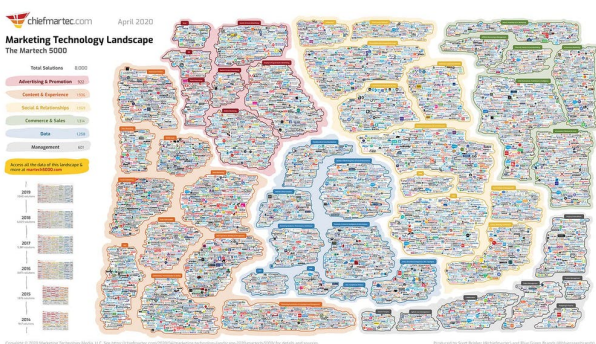

(a) MarTech 5000

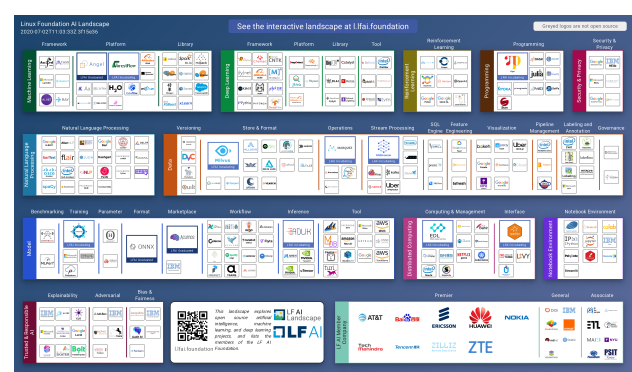

(b) Linux Foundation Landscape

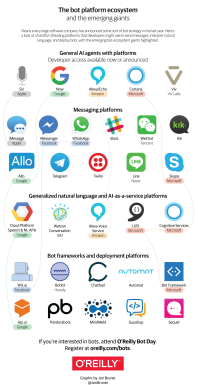

(c) Bot Ecosystem

Figure 1: Sample Logomaps

are graphical symbols commonly used to differentiate brands. Well-designed logos are easily recognizable and considered visual shortcuts to humans. However, for machines extracting this knowledge can be challenge without the right training set. First, a logo can either come in color, black-and-white, or modified color version. Some companies have logos that include a symbol and a text, and sometimes only the symbol or only the text are shown. Logos can come with a light or dark background, perhaps even colored background. Some companies have variation of their logos for different use contexts. Variations could also exist for different groups within a firm (subsidiary, business unit) or a logo could refer to a product, technology or other offering. Logos can also come in rotated form or can be partially covered by other logos or objects. A method that extracts knowledge out of logomaps thus needs to be able to effectively handle the diversity in logomaps design and logo choices.

\section{Methodology}

We propose a human-centered approach to mining logomaps for business ecosystem intelligence. Rather than automatically transitioning from one stage to another, it is guided by both algorithmic rules and human judgment and interventions [22]. In doing so, the approach aims to balance the strengths of humans and machines at each stage [23]. The approach consists of six components (see Figure 2): (1) logomap identification, (2) logo database curation, (3) logomap processing and labeling, (4) knowledge graph construction, (5) knowledge graph visualization, and (6) sensemaking.

\subsection{Logomap Identification}

Logomaps appear in a wide range of sources, including market research websites, analyst reports, investor presentations, and social media posts. However, given the diversity of outlets, there is really no single source that captures them comprehensively. One proven approach to identifying logomaps is to use Google's image search capability, in particular by using the keyword < "industry name" ecosystem or landscape or market>. Over the past few years we have collected and curated 100+ logomaps covering different technology industries and creator groups through this approach as well as monitoring social media and other channels. We have organized all of these logomaps in an interactive web gallery. In addition to the high-resolution image, we have also attempted to capture any available meta-data, such as logomap title, industry and/or context, creator name (individual, firm, investor), and creation date.

\subsection{Logo Database Curation}

To the best of our knowledge, there is no single database that comprehensively captures and tracks logos. There are some sites that specialize in capturing corporate logos (e.g., BrandEPS, RiteKit, Seeklogo) but mainly focus on well-established firms and brands. The WIPO Global Brand Database ${ }^{3}$ is arguably one of the most comprehensive sources, but startups are not well captured here. Another potential source is the USPTO's database of registered trademarks ${ }^{4}$, but automated data extraction is limited. Many ecosystem data providers, such as Crunchbase, Angel.co, Pitchbook, and Owler, have started capturing corporate logos and in particular startups. However, technology, product, and solution logos are often not captured. A Google image search on $<$ entity $\log 0>$ provides useful information but results can vary and are generally not very structured. Logos are also available on the Wikipedia entry of entities, but historical evolution and variations are not captured.

\footnotetext{
${ }^{3}$ https: / / www3.wipo.int/branddb/en/

${ }^{4}$ https: / / www . uspto.gov/trademark
} 


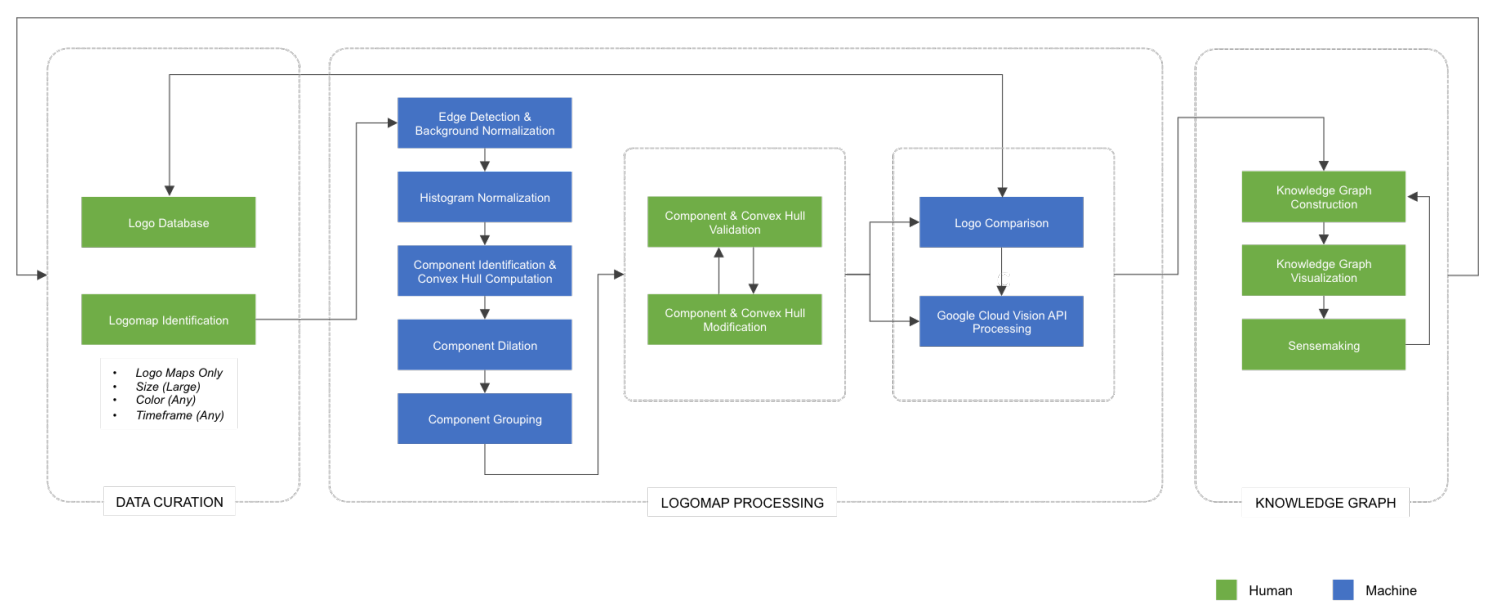

Figure 2: Logomap Mining Methodology.

These observations highlight the challenges of creating a comprehensive logo database for ecosystem intelligence. First, not all entities of interest are captured (e.g., firm, brand, product, etc.). Second, small and emerging entities are not often tracked. Third, there can be many design variations for a given entity. And lastly, logos can evolve over time.

To overcome these issue we used a multi-pronged approach. We first automatically extracted logos from multiple structured data sources. Second, any logo identified in our logomaps that did not match logos from our current database was reversed-searched and mapped using the Google Cloud Vision API, and then added to our database. Lastly, if no matches were found, we allow a human-analyst to manually index them.

\subsection{Logomap Processing and Labeling}

3.3.1. Machine Processing (I) The process begins with the preparation of images for subsequent image mining. The input is a high-resolution image; the output includes a proposed outline of logos that should be "cut" and sent to the Google Cloud Vision API and a detection of hierarchies and labels. All of the boundaries can be manipulated in our logomap mining system.

The first step includes edge detection and background normalization with Scharr filter, which is a type of Sobel Filter with less rotational asymmetry [24]. This is followed by Histogram normalization with Triangle Method, which works by assuming there is a peak near one end of the distribution which is true for this data and produces the best results for a distribution of this type. Next, we find all connected components then calculate their convex hull (the smallest polygon that is convex and still contains the full connected component). Components are then filtered into large and small based on the convex hull. Large components will be assumed to be grouping elements (lines and boxes that separate the logos into groups), while the small components will be assumed to be either logos or labels. We then generate an image that contains only the small components and a separate image that contains only the larger components. Next, we iterate as follows: (1) Dilate the small components, and (2) Subtract the large components from the resulting dilated image. This has the effect of expanding the small components to the point that they start touching and therefore merging, while not allowing the expansion to pass through any large structures which are assumed to be grouping elements. The result is an image that has fewer but larger connected components. We achieve this as follows. Small connected components are merged; components that are distant or separated by a grouping element (such as a line or box) are not merged. The resulting image then represents the grouping of logos and these large connected components describe the extent of each group. Lastly, the small connected components found earlier (that are assumed to be logos or labels) are retrieved and sectioned into groups based on the grouping information found above.

3.3.2. Human Processing Once the image processing algorithms have completed, boundaries, regions, and label and icon entities have been detected. At this stage, human intervention is necessary to inspect, validate, and/or modify the results. Using an 


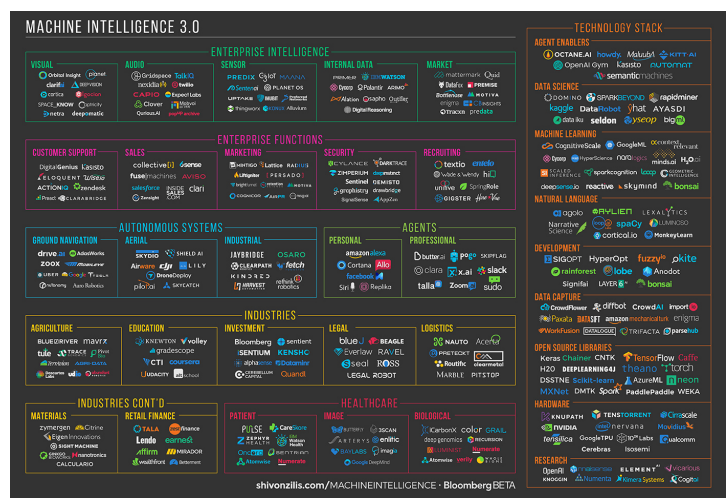

(a) Original Logomap

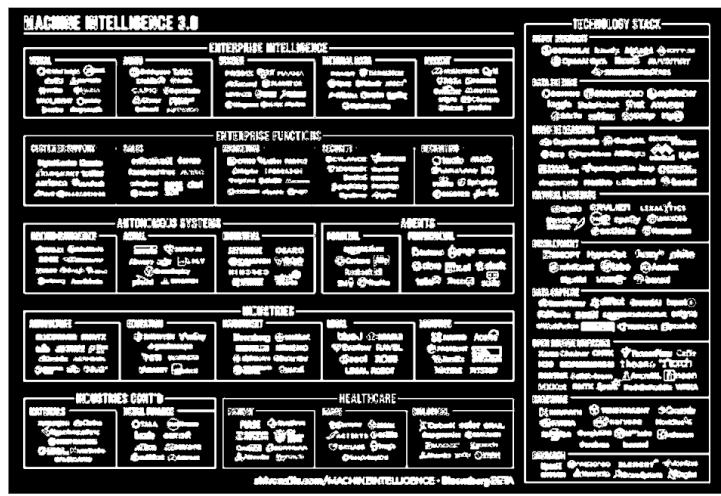

(c) Histogram normalization

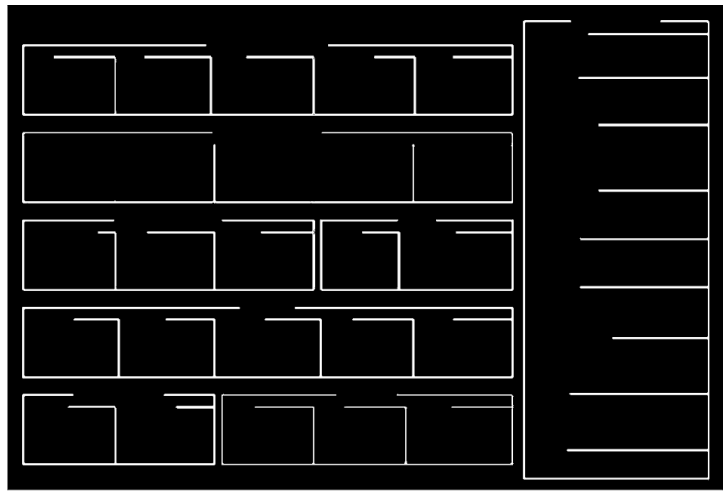

(e) Component dilation I

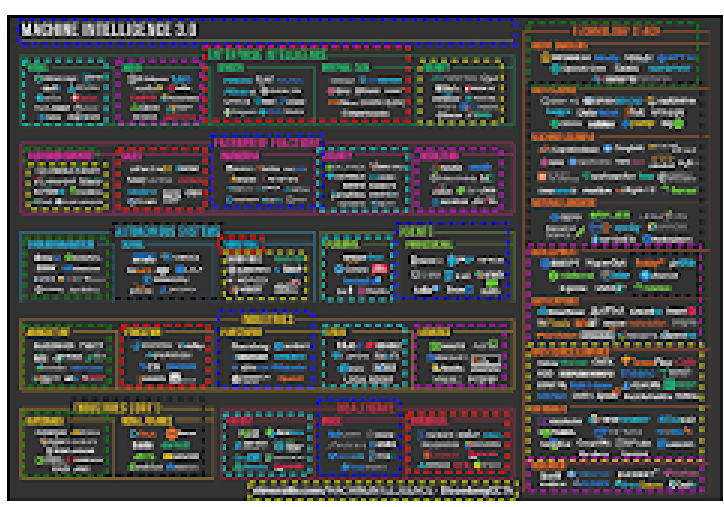

(g) Component grouping I

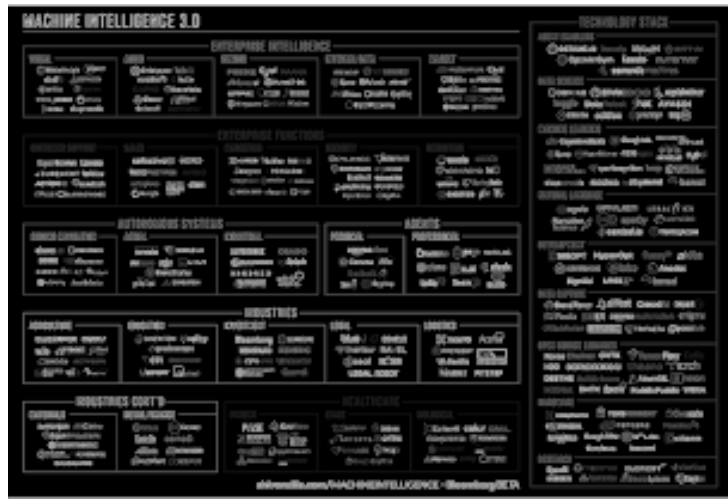

(b) Edge detection and background normalization

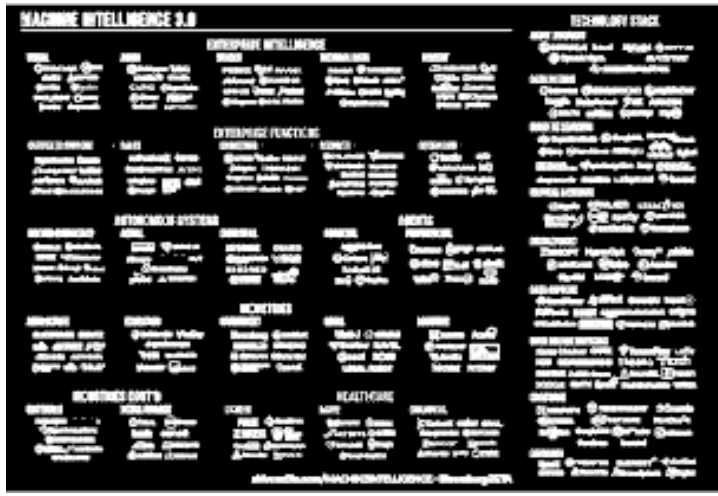

(d) Component identification and convex hull computation

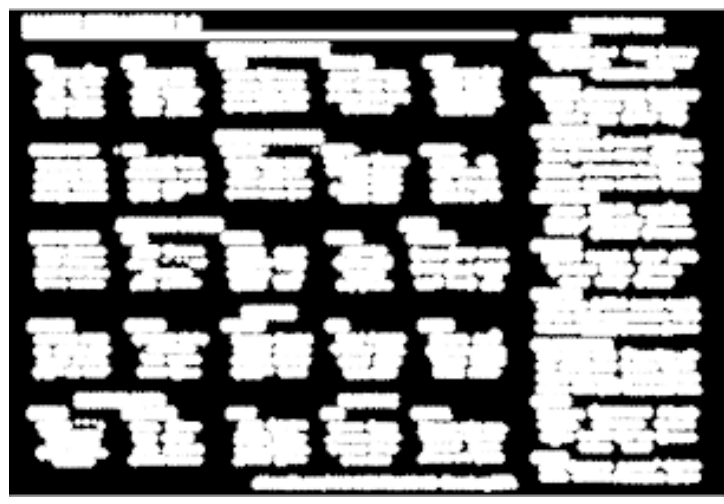

(f) Component dilation II

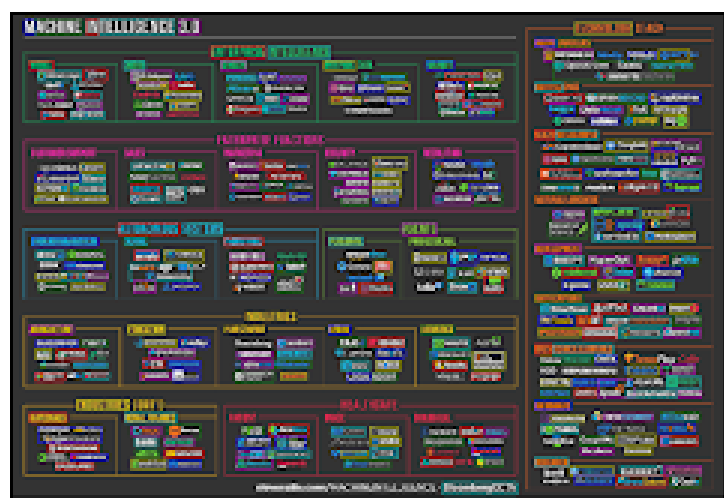

(h) Component grouping II

Figure 3: Image Preparation and Logo/Hierarchy Detection 
(a)
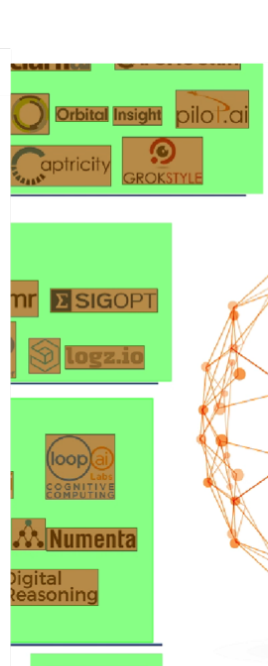

IOT//IOT

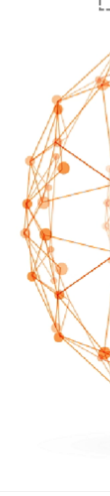

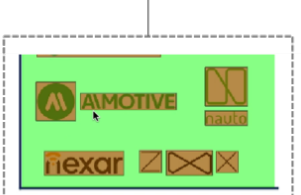

-
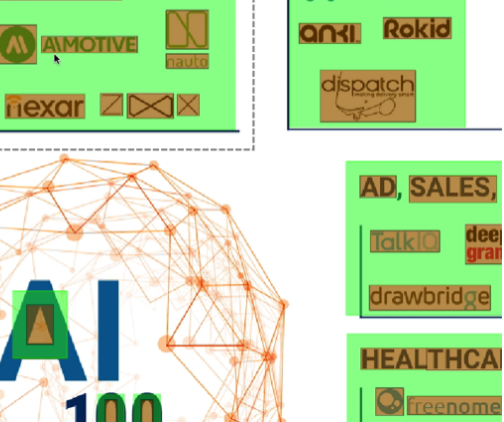

Q6
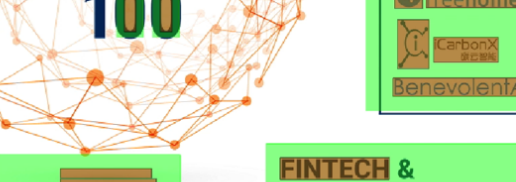

COMMERCE
.

.

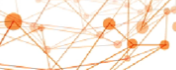

MERC
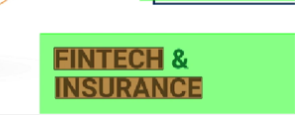

(b)

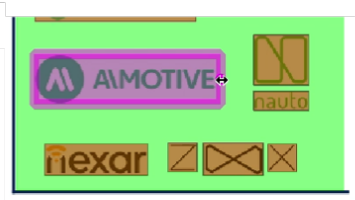

(c)

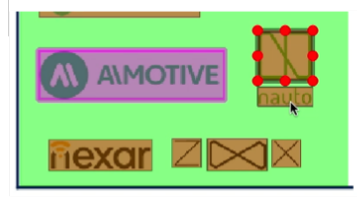

(d)

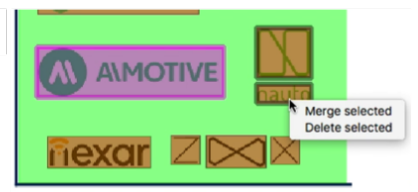

Figure 4: Interactive Processing of Logomaps. (a) Machine-labeled logomap; (b) Extend boundary around logo; (c) Multi-select logos; and (d) Merge logo areas.

interactive tool, the user can specify polygon boundaries that have been missed, combine, integrate, or split icon regions (e.g., logo icon and logo text needs to be combined), labels can be specified (see Figure 4). To do so, users select the boundary tool and modify the shape (either logo boundary or hierarchical boundary) through dragging. To combine items, users shift-select the components and use right-click to select the combine option. Hierarchical layers are created for anything contained within a boundary. The boundary color indicates if the algorithm considered is a label or logo entity. To modify the type of entity, users right-click select the type option. By allowing users to confirm or modify the results of the machine learning algorithm, we provide feedback into the accuracy of the algorithms. The system learns from these updates in its subsequent processing. Moreover, since the range of designs of logomaps is so wide, it is virtually impossible to assume that our algorithms will be perfect. The updated logomap with its respective polygon areas is saved and areas are "cut" for subsequent machine processing.

3.3.3. Machine Processing (II) The logo areas are then compared to the logos in our database. If any logo is present, we capture it. If it is not present, we send the cut polygon area to the Google Cloud Vision API. This returns the label or URLs of any pages where that logo has been uploaded in addition to any text that Google detected in the image. These URLs and text detections are then used to compare logos and labels across multiple logomaps.

\subsection{Knowledge Graph Construction}

There are several potential approaches to construct a knowledge graph of the entities extracted from a logomap. One basic approach is to consider logos without any consideration of the hierarchical boundaries presented in a logomap. With this proximity-based approach, we would create links between logos that fall within a certain distance from each other. A modification of this approach is to consider only connecting logos that fall within a grouping boundary. With this group-based approach all logos contained within a border are then connected; logos within a certain thresholded distance from other logos are also connected. However, with this uni-partite approach we not only lose information about the labels used within the logomaps but it also complicates the what to do with logos that appear in multiple maps. Furthermore since labels and hierarchical boundaries are of interest, we instead opted for a bi-partite network construction approach, with both logo and label nodes. With this approach we link logo-to-label and label-to-label nodes if there are multiple levels of hierarchies. This approach also allows the consideration of linking logos across multiple logomaps. 


\subsection{Knowledge Graph Visualization}

Given the complex interconnected nature of our knowledge graph, we opted to use a force-directed network algorithm to position in nodes spatially. We used the force-directed Yifan Hu multi-level layout algorithm with default parameters [25] to position nodes. A force-based layout is based on the idea that network entities are shaped by mechanical laws, assigning repulsive forces between nodes and attraction forces between endpoints of edges. The use of a force-based layout is particularly appealing when the motivating issue is to identify central or prominent nodes, peripheral actors, or clusters in an ecosystem. To ensure readability and aesthetics, we followed several visual design principles [26]. The node size is proportional to an entity's importance in the knowledge graph as measured by its Betweenness centrality. To gain insight into the presence of subcommunities, we color encode nodes with the corresponding modularity class. We used a NoOverlap algorithm to space out nodes and address potential visual occlusion issues.

\subsection{Sensemaking}

The ultimate goal of our knowledge graph visualization is not to create a aesthetic graphical depiction, but rather to provide a platform for human insight and foresight, helping to identify trends, patterns, and outliers [17]. Sense-making has its roots in cognitive psychology and many different models have been developed [27]. The consensus across these models is that the sense-making process is cyclic and interactive, involving both discovery and creation. During the generation loop an individual searches for representations. In the data coverage loop, we instantiate these representations. Based on these insights, we shift our representation and begin again. Together this forms a complete sense-making loop. The visualization of knowledge graphs embedded across multiple logomaps can therefore be seen to support the ecosystem intelligence sense-making process. Through these knowledge graph visualizations we seek to uncover confirmation, inconsistencies, and possible "aha" moments. If confirmation is not achieved, we return to develop alternative visualizations or specify new boundaries.

\section{Use Case}

We demonstrate the logomap mining method in the context of the artificial intelligence (AI) ecosystem. AI is a rapidly emerging field that encompasses several different technologies, impacts almost all industries, and is of strategic importance to many countries $[28,29]$. Consequently, we would expect many stakeholders interested in the structure and dynamics of the underlying ecosystem. Given that it is still in an immature state, we would also expect different focus areas and perspectives. We begin by identifying different logomaps on the AI ecosystem through an initial and related Google image search through salient keywords ("AI ecosystem", "AI market", "AI landscape"). The forward and backward search leads to many results, including some logomaps of higher and lower and higher quality. We constrain the sample of logomaps to high-resolution images only to ensure effective identification of logos. We eliminate any duplicates and only include the latest version of logomaps with multiple editions. This led to the identification of 26 logomaps. A sample of these are shown in Figure 5.

Next, we apply the multiphase machine algorithms to detect edges, filter, and identify logos and labels. Manual manipulation of label and logo boundaries are conducted for each logomap. Once completed, logos are extracted. We compare the logos to the logo database we curated from entries in Crunchbase, Angel.co, Owler, and other ecosystem data sources. Those that have a match are indexed and saved. Those that are not identified are sent to the Google Cloud Vision. Logo labels are returned. We then use the hierarchical groupings along with the logo information to create a bi-partite graph of label and logo nodes. We compute Betweennesss centrality scores and modularity class for each node. Next we visualize the network using the force-directed layout and color encode and size nodes with the metrics above.

The resulting ecosystem visualization is shown in Figure 6. Manual inspection of the color-encoded communities reveals clusters associated with healthcare, analytics, and finance. An interesting observation is that out of the 3,000+ identified only 487 appear across multiple logomaps, suggesting only a small core of key companies and divergent perspectives by industry and countries. This may imply that there are many niche AI firms. The visualization also reveals a dense core and periphery structure with some more disconnected clusters. Relative proximity of the tools/platforms/enablers and infrastructure clusters highlights the closeness of these two segments.

\section{Discussion and Future Work}

Our case study on AI logomaps provides a first demonstration of how to mine ecosystem knowledge from a set of image-based data. However, as with 


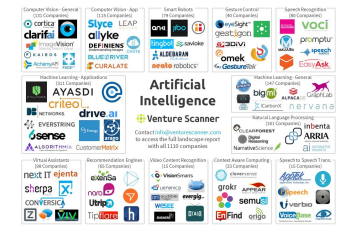

(a) Venture Scanner

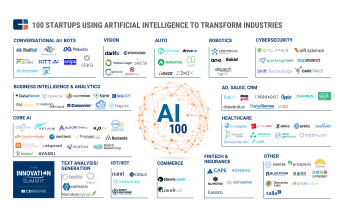

(b) CB Insights

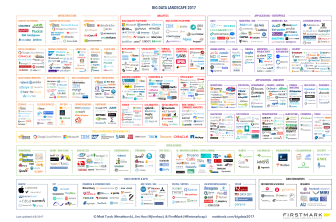

(c) Firstmark Capital

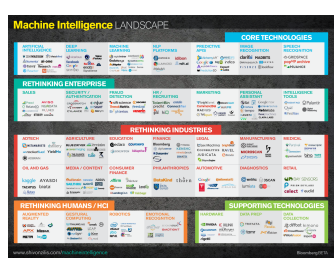

(d) Bloomberg BETA

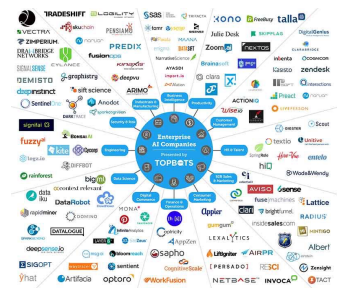

(e) TopBots

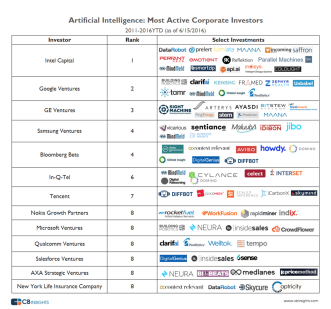

(i) CB Insights

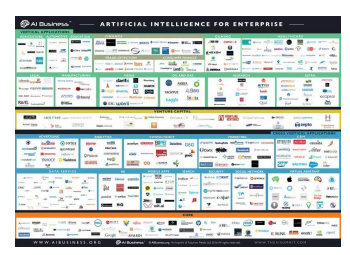

(f) AIBusiness.org

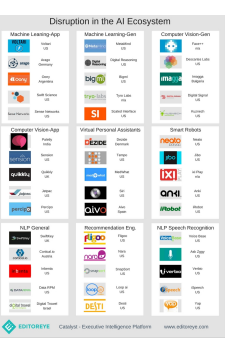

(j) EditorEye

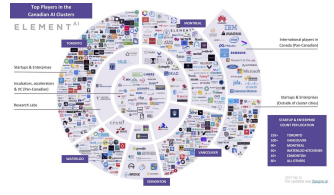

(g) ElementAI

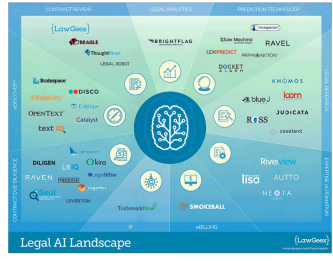

(k) LawGeex

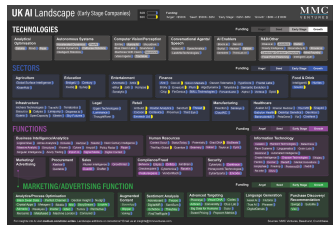

(h) MMC Ventures

Figure 5: Illustrative Logomaps of the AI Industry

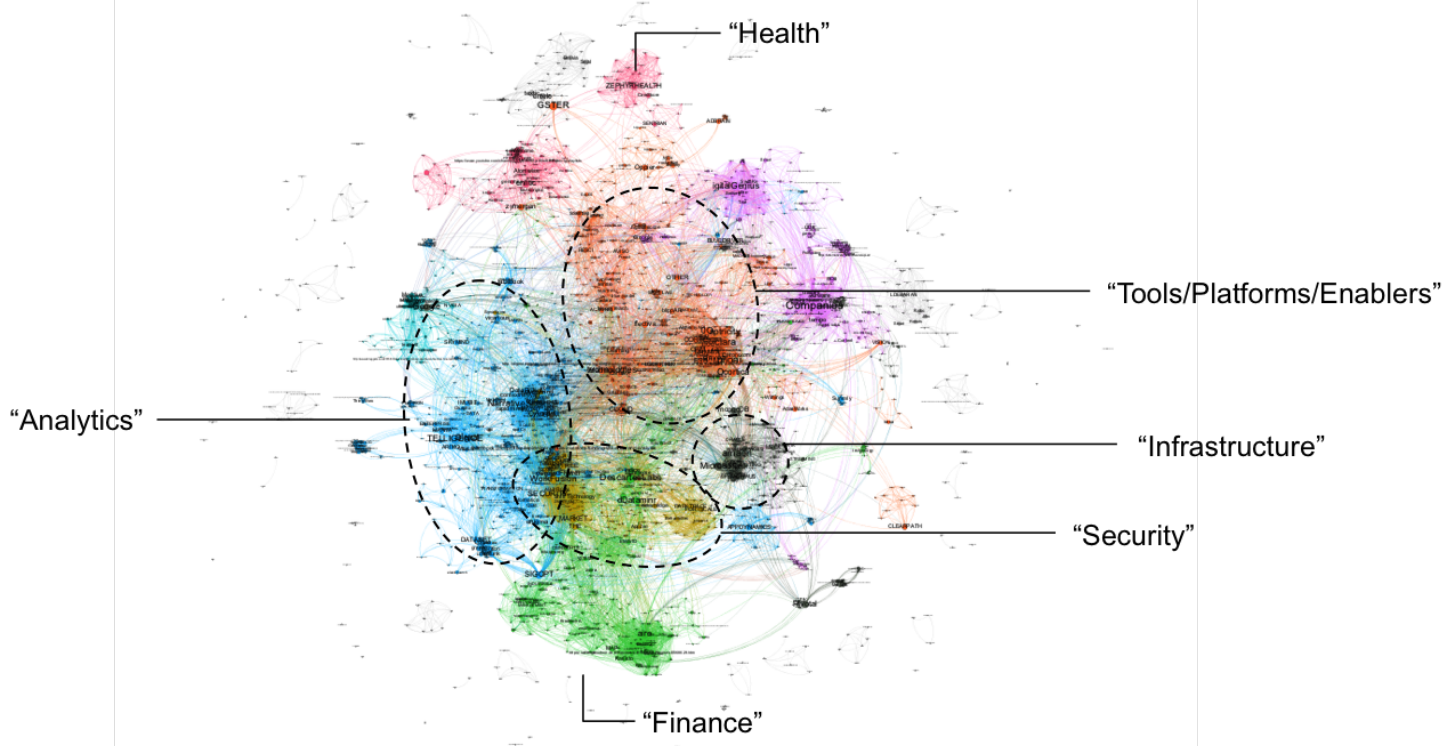

Figure 6: The Structure of the AI Ecosystem derived from 26 Logomaps. 
all data-driven research initiatives, the novelty and scope of insights generated largely depends on the quality of the underlying data. For our context, we curated what we believe is a fairly comprehensive set of logomaps about AI over the past few years. It includes a consideration of $\mathrm{AI}$ in specific industries (e.g., finance, healthcare, agriculture, etc.) and countries (e.g., Germany, France). Our results are likely going to be skewed towards the logomap contexts that are included. As more logomaps and logomap contexts are included, the more fine grained and comprehensive our results will likely get. Nevertheless, we show that even a potential subset of logomaps can provide insights into patterns and trends, including the commonality of a core set of firms, disjoint consideration and exclusion of a large chunk of the ecosystem, and clustering of market segments. At the same time our approach reveals a number of challenges and opportunities associated with the analysis of logomaps and raises other considerations that will have to be made to make the process and insights more robust, human-centered, and scalable.

\subsection{Data Complementarity}

One of the key assumptions we make is that image-based data may offer some novel knowledge that is not contained in other data sources. While we demonstrate what knowledge can be contained in image-based data, we do not explicitly show how image-based data differs from other structured data. A future research study should thus investigate in what ways image-based data may be different from traditional sources, perhaps in the type of knowledge that is embedded in them (such as hierarchy or content), the contexts it examines (e.g., industries, technologies, types of firms), or what information it may explicitly link (e.g., companies and technologies, companies and segments, etc.). In doing so, we would be able to comment not only on similarity and differences, but also on the value complementarity of image-based data. As identified in previous work, a single data source is not likely sufficient for comprehensive ecosystem intelligence. Instead, multiple, linked data sources can provide complementary, triangulated insights.

\subsection{Multiple Perspectives}

Logomaps are created by many different types of actors, including market research firms, financial institutions and investors, professional and non-profit associations, industry analysts, and academic researchers. An interesting future analysis would be to compare how different stakeholder groups view the same underlying ecosystem. Do investors and market research analysts focus on the same firms and segments? Do they label segments differently? Do they group ecosystem actors differently? The comparison of perspectives can not only reveal potentially different lenses, highlighting common and different focus areas, but also inform different industry analysis strategies.

\subsection{Temporal Evolution}

Ecosystems are inherently dynamic; firms enter, exit, merge, get acquired, shift to new segments, and so on. A temporal consideration of the underlying structure is thus essential to good ecosystem intelligence. Logomaps represent the creator's assessment of an ecosystem at a given point in time. An important extension of this work thus would include the consideration of this temporal data and examine how the thinking has evolved. While some logomaps provide dates directly on the image, images sometimes also have a date and time stamp associated with it.

\subsection{Automated Summaries}

To improve the user experience, it would be valuable to not just provide a visual representation of the ecosystem, but also automated statistical summaries of individual and collective logomaps. For each logomap, useful metrics could include the number of logos, number of unique logos, number of groups, number of logos/group, and textual description of hierarchy. Similarly, it would be beneficial to provide aggregate statistical summaries of multiple logomaps, such as average number of logos in each logomap, number of unique logos, average number of groups in each logomap, and average number of logos/group. What would enhance automated summaries even further is to combine these summaries with other data, such as firm performance (revenue, profit), investment data (number of investments, total funding amount) or innovation data (patents, R\&D).

\subsection{Alternative Knowledge Graph Visualizations}

Given that we were interested in the structure of an ecosystem, a node-link based network visualization was clearly the most logical. However, a force-directed layout is not the only choice. Grouped layouts, using either physics-based or circular layouts may also provide important insights into the grouping and clustering of an ecosystem. Another potential option may include a nested tree structure. Alternative representations need to be explored. 


\subsection{Cluster Labeling}

Cluster labeling was done manually. As an extension, text analytic methods can be used determine cluster topics/labels from company descriptions.

\section{Concluding Remarks}

In this study we presented a method and human-centered AI-based tool to extract ecosystem intelligence from one type of image-based data, namely logomaps. An illustrative case study reveals commonalities between logomaps, but within a single image many intelligence blindspots exist. Significant new ecosystem insights can be generated when leveraging multiple logomaps. We hope that our study stimulates future work in mining highly unstructured data for ecosystem intelligence.

\section{Acknowledgments}

The author thanks Peter Presti, Jeff Wilson, and Tyler Labean for contribution to tool design and development.

\section{References}

[1] M. Iansiti and R. Levien, The keystone advantage: what the new dynamics of business ecosystems mean for strategy, innovation, and sustainability. Harvard Business Press, 2004.

[2] R. Kapoor, "Ecosystems: Broadening the locus of value creation," Journal of Organization Design, vol. 7, no. 1, p. 12, 2018.

[3] D. J. Teece, "Business ecosystem," The Palgrave Encyclopedia of Strategic Management, pp. 151-154, 2016.

[4] R. Adner, J. E. Oxley, and B. S. Silverman, Collaboration and competition in business ecosystems. Emerald Bingley, 2013.

[5] R. C. Basole, "Visual business ecosystem intelligence: Lessons from the field," IEEE Computer Graphics and Applications, vol. 34, no. 5, pp. 26-34, 2014.

[6] R. C. Basole, T. Clear, M. Hu, H. Mehrotra, and J. Stasko, "Understanding interfirm relationships in business ecosystems with interactive visualization," IEEE Transactions on Visualization and Computer Graphics, vol. 19, no. 12, pp. 2526-2535, 2013.

[7] R. C. Basole, A. Srinivasan, H. Park, and S. Patel, "ecoxight: Discovery, exploration, and analysis of business ecosystems using interactive visualization," ACM Transactions on Management Information Systems (TMIS), vol. 9, no. 2, pp. 1-26, 2018.

[8] B. R. Iyer and R. C. Basole, "Visualization to understand ecosystems," Communications of the ACM, vol. 59, no. 11, pp. 27-30, 2016.

[9] R. C. Basole, "Understanding ecosystem data," in Proceedings of the 53rd Hawaii International Conference on System Sciences, pp. 5718-5727.
[10] D. Delen, Real-world data mining: Applied business analytics and decision making. FT Press, 2014.

[11] J. F. Moore, "Predators and prey: a new ecology of competition," Harvard Business Review, vol. 71, no. 3, pp. 75-86, 1993.

[12] R. Adner, "Ecosystem as structure: An actionable construct for strategy," Journal of Management, vol. 43, no. 1, pp. 39-58, 2017.

[13] M. G. Jacobides, C. Cennamo, and A. Gawer, "Towards a theory of ecosystems," Strategic Management Journal, vol. 39, no. 8, pp. 2255-2276, 2018.

[14] K. Järvi, S. Kortelainen, et al., "Taking stock of empirical research on business ecosystems: a literature review," International Journal of Business and Systems Research, vol. 11, no. 3, pp. 215-228, 2017.

[15] R. C. Basole, J. Huhtamäki, K. Still, and M. G. Russell, "Visual decision support for business ecosystem analysis," Expert Systems with Applications, vol. 65, pp. 271-282, 2016

[16] A. Shipilov and A. Gawer, "Integrating research on interorganizational networks and ecosystems," Academy of Management Annals, vol. 14, no. 1, pp. 92-121, 2020.

[17] M. Card, Readings in information visualization: Using vision to think. Morgan Kaufmann, 1999.

[18] C. Steger, M. Ulrich, and C. Wiedemann, Machine vision algorithms and applications. John Wiley \& Sons, 2018.

[19] R. Szeliski, Computer vision: Algorithms and applications. Springer Science \& Business Media, 2010.

[20] P. W. Henderson and J. A. Cote, "Guidelines for selecting or modifying logos," Journal of Marketing, vol. 62, no. 2, pp. 14-30, 1998.

[21] M. Wertheimer, Gestalt theory, ch. 1, pp. 1-11. Kegan Paul, Trench, Trubner \& Company, 1938.

[22] A. Endert, W. Ribarsky, C. Turkay, B. W. Wong, I. Nabney, I. D. Blanco, and F. Rossi, "The state of the art in integrating machine learning into visual analytics," in Computer Graphics Forum, vol. 36, pp. 458-486, Wiley Online Library, 2017.

[23] P. R. Daugherty and H. J. Wilson, Human+ machine: reimagining work in the age of AI. Harvard Business Press, 2018.

[24] G. Shrivakshan and C. Chandrasekar, "A comparison of various edge detection techniques used in image processing," International Journal of Computer Science Issues (IJCSI), vol. 9, no. 5, p. 269, 2012.

[25] Y. Hu, "Efficient, high-quality force-directed graph drawing," Mathematica Journal, vol. 10, no. 1, pp. 37-71, 2005.

[26] C. Bennett, J. Ryall, L. Spalteholz, and A. Gooch, "The aesthetics of graph visualization.," Computational Aesthetics, vol. 2007, pp. 57-64, 2007.

[27] C. North, "Toward measuring visualization insight," IEEE Computer Graphics and Applications, vol. 26, no. 3, pp. 6-9, 2006.

[28] R. Perrault, Y. Shoham, E. Brynjolfsson, J. Clark, J. Etchemendy, B. Grosz, T. Lyons, J. Manyika, S. Mishra, and J. C. Niebles, "The AI index 2019 annual report," AI Index Steering Committee, Human-Centered AI Institute, Stanford University, Stanford, CA, 2019.

[29] M. Iansiti and K. Lakhani, Competing in the Age of Artificial Intelligence. Harvard Business Press, 2020. 